\title{
VALIDATION AND DEPLOYMENT OF THE FIRST LIDAR BASED WEATHER ObSERVATION NeTWORK IN NeW York State: The NYS MesoNet Project
}

\author{
L. Thobois ${ }^{1 *}$, J. Freedman ${ }^{2}$, P. Royer ${ }^{1}$, J. Brotzge $^{2}$, E. Joseph ${ }^{2}$ \\ ${ }^{1}$ Leosphere: Lidar Environmental Observations, Orsay, France, *lthobois@leosphere.com \\ ${ }^{2}$ University of Albany, Albany, USA
}

\begin{abstract}
The number and quality of atmospheric observations used by meteorologists and operational forecasters are increasing year after year, and yet, consistent improvements in forecast skill remains a challenge. While contributing factors involving these challenges have been identified, including the difficulty in accurately establishing initial conditions, improving the observations at regional and local scales is necessary for accurate depiction of the atmospheric boundary layer (below $2 \mathrm{~km}$ ), particularly the wind profile, in high resolution numerical models. Above the uncertainty of weather forecasts, the goal is also to improve the detection of severe and extreme weather events (severe thunderstorms, tornadoes and other mesoscale phenomena) that can adversely affect life, property and commerce, primarily in densely populated urban centers.
\end{abstract}

This paper will describe the New York State Mesonet that is being deployed in the state of New York, USA. It is composed of 126 stations including 17 profiler sites. These sites will acquire continuous upper air observations through the combination of WINDCUBE Lidars and microwave radiometers. These stations will provide temperature, relative humidity \& "3D" wind profile measurements through and above the planetary boundary layer (PBL) and will retrieve derived atmospheric quantities such as the PBL height, cloud base, momentum fluxes, and aerosol $\&$ cloud optical properties. The different modes and configurations that will be used for the Lidars are discussed. The performances in terms of data availability and wind accuracy and precision are evaluated. Several profiles with specific wind and aerosol features are presented to illustrate the benefits of the use of Coherent Doppler Lidars to monitor accurately the PBL.

\section{INTRODUCTION}

Despite the constant effort to improve weather forecasts over the last few decades, depiction of the evolution of the planetary boundary layer (PBL) remains a challenge. Today, there is a growing interest focusing on local and regional scales. Indeed, for severe weather, weather forecasts are crucial to drastically reduce the impact on human lives and economical activities where weather risk exposure is the highest (urban, business, industrial area). The forecast goal is to provide information regarding weather risk to support local authorities and decision makers. To reach this objective, numerical weather prediction (NWP) models must be coupling increased spatial resolution with improved data assimilation methods. These models, however, require more comprehensive local observations (e.g. the 3D wind field) as initial conditions. But existing weather observation networks were designed for national coverage and not for providing dense observations to high resolution models. New sensors are then required to provide accurate $3 \mathrm{D}$ observations with high spatial resolution from the surface through the top of the PBL. Wind Lidar technologies developed since the 1990s have expanded vastly in the 2000s with the introduction of commercial off-the-shelf fiber optics components developed for the telecommunication industry. This Lidar technology now incorporates cost effective sensors thanks to industrial manufacturing. Today, Coherent Pulsed Doppler Lidar (CDL) is being evaluated globally in a wide variety of projects to determine its benefits for weather observing networks [1][2].

In this paper, the incorporation of CDL into the operations of the New York State Mesonet (NYSM) is presented. The capabilities of CDLs used in this network are described in terms of wind and aerosol/cloud measurements. Their potential benefits for future observing networks 
are demonstrated with a few examples of wind and aerosol cases.

\section{THE NEW YORK STATE MESONET}

In 2014 Governor Cuomo, with funding from the Federal Emergency Management Agency, initiated the New York State Early Warning Weather Detection System. The centerpiece of this system is the NYSM, an advanced, statewide weather station network explicitly designed to enhance local data collection for improved local weather monitoring and prediction. It consists of a network of 126 weather stations that provide surface temperature, relative humidity, wind speed and direction, precipitation, solar radiation, atmospheric pressure, photographic images and soil moisture and temperature at three depths (5, 25 , and $50 \mathrm{~cm}$ ). In addition, seventeen of the sites are being outfitted with WINDCUBE coherent Doppler lidars (CDL), Microwave radiometers and sun photometers providing 3D wind, turbulence, temperature, and moisture profiles in the vertical within and above the PBL, and cloud and aerosol information, as well as mixing layer and residual layer height. Upon completion in 2017, real time data along with graphical products will be available to the public via a website (http://nysmesonet.org).

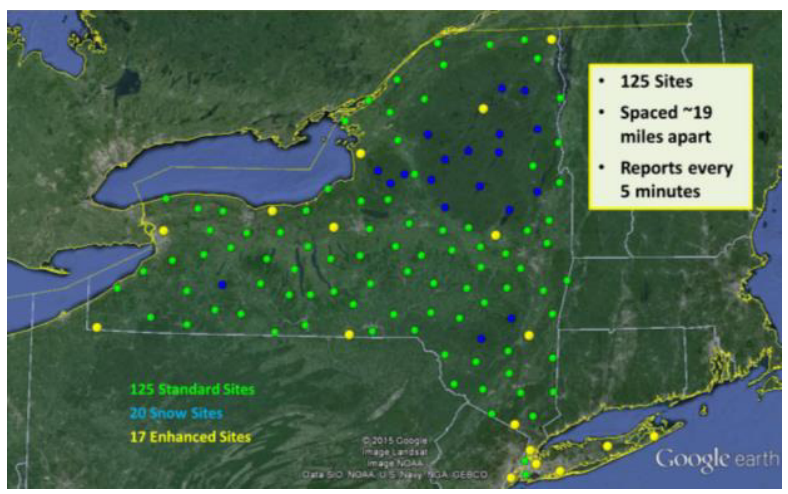

Figure 1 NYS MESONET deployment

\section{PROFILER STATIONS WITH CDLS}

Thanks to the rapid development of fiber based Lidar technology, more than a thousand of CDLs are now in use worldwide as operational instruments for wind energy, meteorology and air traffic applications. CDLs have been chosen for the NYSM to provide one of the most important parameters to be assimilated into NWP model wind profiles. The CDLs of the NYSM are LEOSPHERE WINDCUBE100S scanning CDLs that are configured in Doppler Beam Swinging (DBS) scenario to provide vertical profiles of horizontal wind speeds, vertical wind speed and wind direction from the ground up to the top of the PBL $(1-2 \mathrm{~km})$. The physical resolution chosen is $25 \mathrm{~m}$ corresponding to pulse duration of $100 \mathrm{~ns}$ and Doppler spectra discretized by 32 points. The accumulation time is $1 \mathrm{~s}$, and the update rate of final products is $5 \mathrm{~min}$. In addition, the signal reflectivity of CDLs will be used to provide aerosol and cloud functionalities: detection of aerosol/cloud layers, retrieval of PBL heights, and backscatter coefficient [3][4].

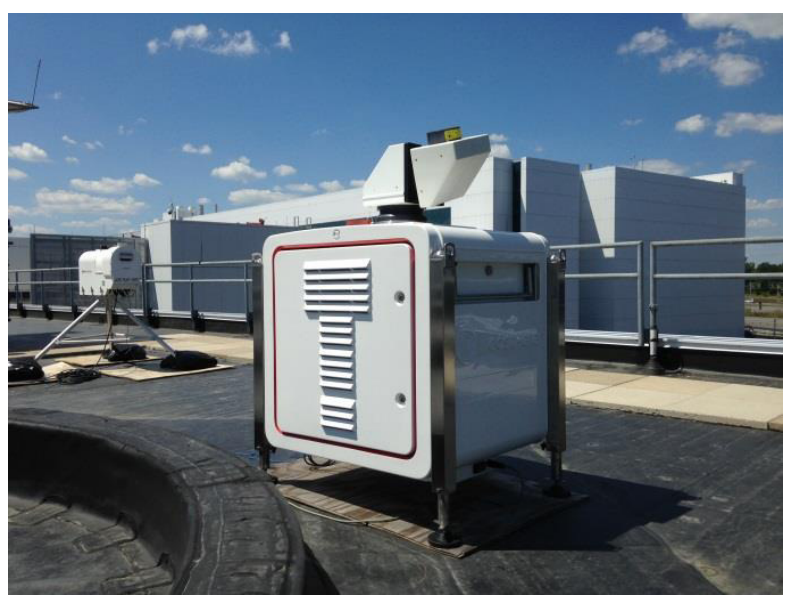

Figure 2 - Installations of WINDCUBE100S lidar and MP3000a radiometer at University of Albany

\section{PRELIMINARY RESULTS}

\section{Monitoring the PBL with CDLs}

As an illustration, the measurements performed by one WINDCUBE100S CDL during two summer days with moderate convection and few clouds and aerosols layers above the boundary layer are presented on Figure 3. The observations show rapid changes in vertical velocity during the late morning and afternoon (local time $~ 1400-1800$ UTC) - evidence of convective plumes and corresponding subsidence) and significant variations of wind speed on these two days. The vertical wind speed allows monitoring the convection from the surface and up to the top of the mixing layer. The backscatter coefficient allows the determination of different vertical layers including the residual layer of the boundary 
layer, clouds and other aerosol layers. In the case of relatively thin cloud layers, the base and top of the multiple cloud layers can be determined.
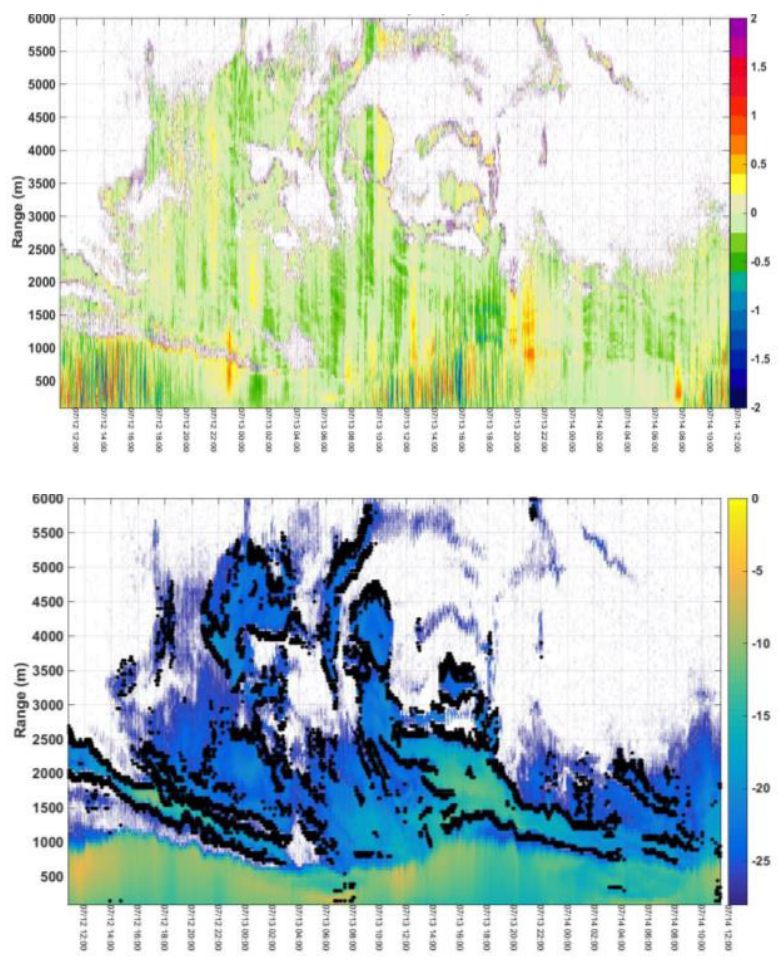

Figure 3 Vertical wind speed (top) and Lidar signal with detected layers (bottom) measured by a CDL

\section{Comparison of CDLs with Radiosondes}

The CDLs that will compose the NYS Mesonet are evaluated in terms of wind accuracy against radiosondes launched twice daily (0000 and 1200 UTC) at the National Weather Service Forecast Office in Albany, NY. Figure 4 shows one example showing excellent agreement between the CDL and radiosonde-derived horizontal wind speed profiles for the 26 August 2015. When evaluating the statistical accuracy and precision of CDL with radiosonde, excellent correlation is obtained in the higher part of the boundary layer (above 500m; Figure 5 (bottom)) with less agreement at lower levels due to turbulence induced by the presence of nearby buildings.

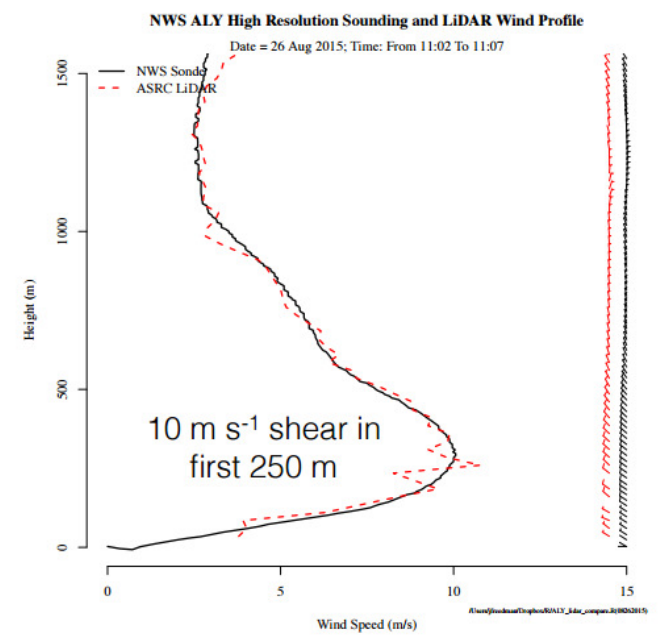

Figure 4 Vertical wind speed (top) and Lidar signal with detected layers (bottom) measured by a CDL
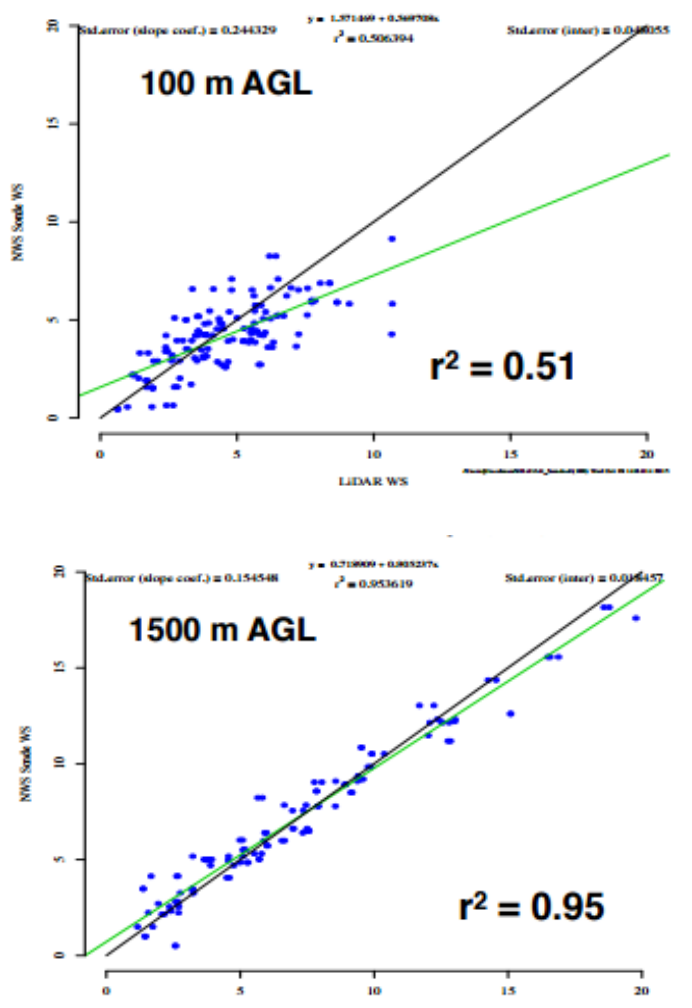

Figure 5 Comparison of horizontal wind speeds between CDL and radiosondes at 100m (top) and $1500 m$ (bottom)

The CDL can also be used to observe rapid changes in the wind speed profile (Figure 6), a characteristic of special interest to the PBL community (e.g., the evolution of low level jets) and the aviation industry (timely detection of changes in low level wind shear). 


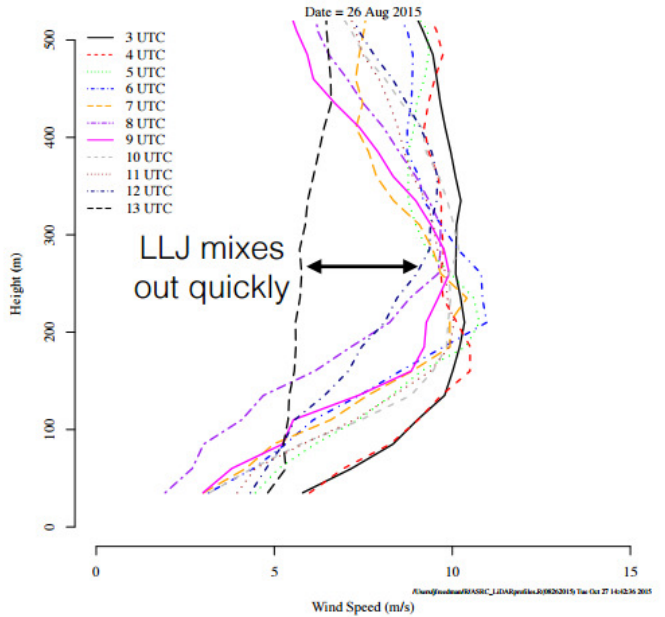

Figure 6 Evolution of wind profiles during one day with a low level jet

\section{Comparison between CDLs}

The CDLs of the NYS Mesonet are also compared with each other to validate the consistency of their data and ensure unbiased measurements at the different sites of the network. Wind speed, Lidar signal strength and data availability are the main parameters that are studied. As an example, wind speed profiles of three co-located CDLs show very good agreement (Figure 7). Advanced intercomparison studies are currently on-going.

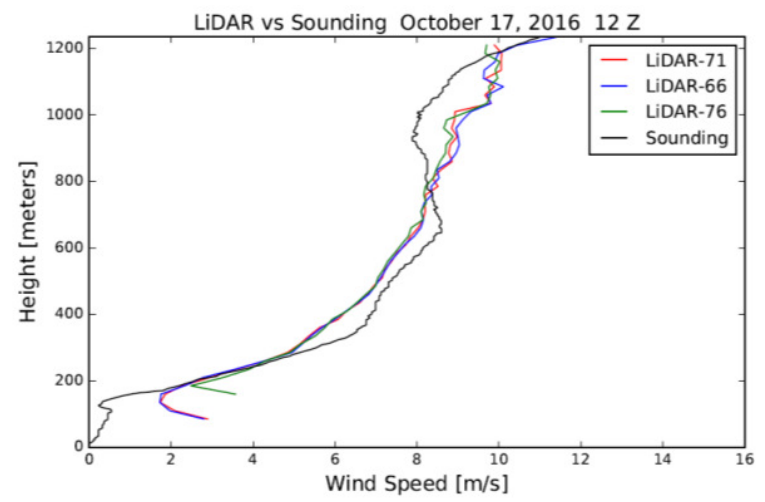

Figure 7 Comparison of wind profiles during one day for three CDLs with the same configuration

\section{CONCLUSIONS}

For the first time, a regional network equipped with CDLs is currently under deployment in the state of New York. The CDLs will be used as a multi-function sensor to provide wind and turbulence measurements but also aerosol and cloud observations usually performed by ceilometers or aerosol Lidars. The measurements of each CDL will be validated in terms of accuracy and precision and compared with a reference CDL. Advanced studies are currently on-going to evaluate their performance and data reliability. Data and derived products from CDLs will be used to improve weather analysis and prediction in combination with MW radiometers that allows the continuous tropospheric profiling of wind, temperature and humidity. A focused effort will be made to assimilate conventional data and derived products like PBL and turbulence into high resolution weather forecast models (NWP) to improve local forecasts.

\section{References}

[1] Illingworth, A. et al., Exploiting Existing Ground-Based Remote Sensing Networks to Improve High-Resolution Weather Forecasts, Bulletin of the American Meteorological Society 2015 96:12, 2107-2125

[2] Wulfmeyer, V. et al., A review of the remote sensing of lower tropospheric thermodynamic profiles and its indispensable role for the understanding and the simulation of water and energy cycles, Rev. Geophys., 53, 819-895, doi:10.1002/2014RG000476.

[3] O'Connor, E. et al.: Observing wind, aerosol particles, cloud and precipitation: Finland's new ground-based remote-sensing network, Atmos. Meas. Tech., 7, 1351-1375, doi:10.5194/amt-71351-2014, 2014.

[4] Hardesty, R. et al., One Year of Doppler Lidar Observations Characterizing Boundary Layer Wind, Turbulence, and Aerosol Structure During the Indianapolis Flux Experiment. In AGU Fall Meeting Abstracts (Vol. 1, p. 03). 